\title{
Precapsular film on the aging human lens: precursor of pseudoexfoliation?
}

Department of University of New York Health Science Center at Syracuse, Syracuse, New York 13210, USA

A J Dark

Department of Pathology B W Streeten

Correspondence to:

Dr B W Streeten, Department of Pathology, Weiskotten of Pathology, Weiskotten Hall, SUNY Health Science Cyracuse, New York 13210, USA.

Accepted for publication 21 June 1990 Ophthalmology, State

Anthony J Dark, Barbara W Streeten

\begin{abstract}
In many older patients we observed a layer of subtle opacification on the anterior lens capsule, appearing as a ground glass film biomicroscopically. This precapsular film (PCF) could be uniform but often had radial grey lines in the mid zone, holes in the paracentral region, and was occasionally rolled up in strings. Lens capsular material obtained at cataract extraction was studied in patients with and without the film. By scanning electron microscopy the PCF appeared as a friable, incomplete fibrillar layer, with rolling of the edges suggesting loose attachment. Ultrastructurally its component fibrils were from 3$6 \mathrm{~nm}$ in diameter, similar to the finer fibrils in pseudoexfoliation (PSX) material. Like PSX material the layer stained positively for the elastic microfibril-associated protein, fibrillin, in a lens with radial striations. These similarities suggested that the two conditions have some relationship and that the PCF may be a precursor of PSX. Finding patches of the fibrillar network in some control patients imples that the PCF is common in patients of cataract age, though seldom detected clinically.
\end{abstract}

Most authors tacitly assume that the classical bull's eye or sunburst appearance of frosting on the anterior lens capsule in pseudoexfoliation (PSX) appears suddenly, fully developed, and

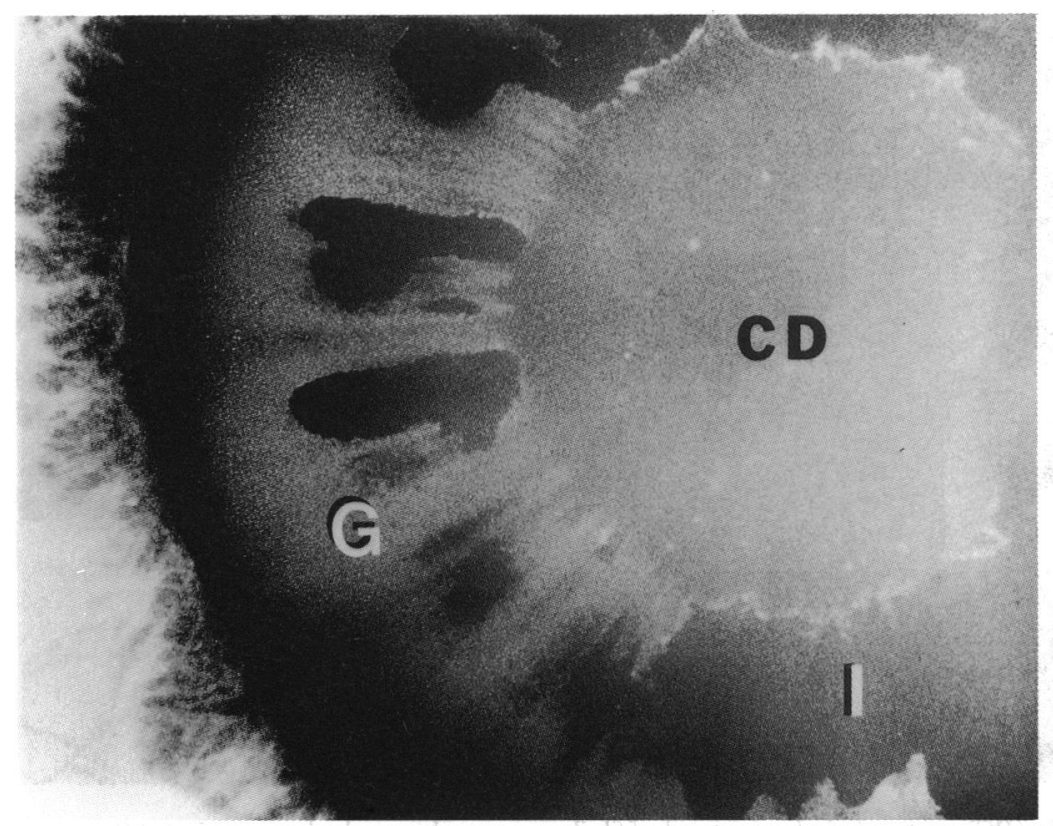

Figure 1 Anterior lens surface in established pseudoexfoliative disease by slit lamp. $C D=$ central disc, $G=$ granular vegetative zone, $I=$ intermediate zone.

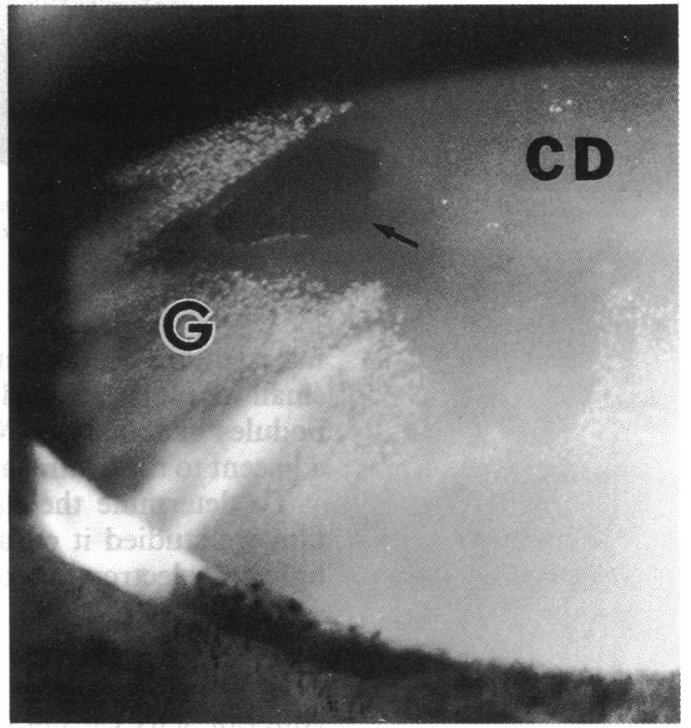

Figure 2 Anterior lens in pseudoexfoliative disease showing white granular zone $(G)$ lying on a homogenous surface film with a paracentral erosion (arrow). $C D=$ central disc.

thereafter undergoes little change (Fig 1). The early biomicroscopic changes are not well recognised and have seldom been reported. ${ }^{12}$ It is difficult to account for this deficit except to speculate that these changes may be of such transience and subtlety that they are seldom observed and variously interpreted. Bartholomew $^{1}$ considered that grey striae on the mid zone of the anterior lens capsule were the earliest biomicroscopic change, the 'pregranular' stage, followed by deposition of vegetations or granules (Busacca's nodules). Evidence for this hypothesis included unilateral cases of PSX associated with striae in the contralateral eye, sector deposition of granules on the striae in some eyes, and a decreasing frequency of striae with age, coinciding with an increase in the granular or established phase.

In a slit-lamp study of patients with and without PSX observed over several years we have noted that the PSX vegetations in some appeared to be deposited on a diffuse but mildly opacified surface layer which sometimes showed focal erosions (Fig 2). A small group of patients in the same age range but without PSX vegetations had a similar ground-glass film on the anterior lens surface, along with other characteristics leading us to hypothesise that the film might be the earliest stage of PSX, before the deposition of granules. This precapsular film (PCF) was a very subtle opacification which could be uniform over the anterior lens or more dense centrally. At least half also had peripheral grey striae similar to those described by Bartholomew,' though he did 

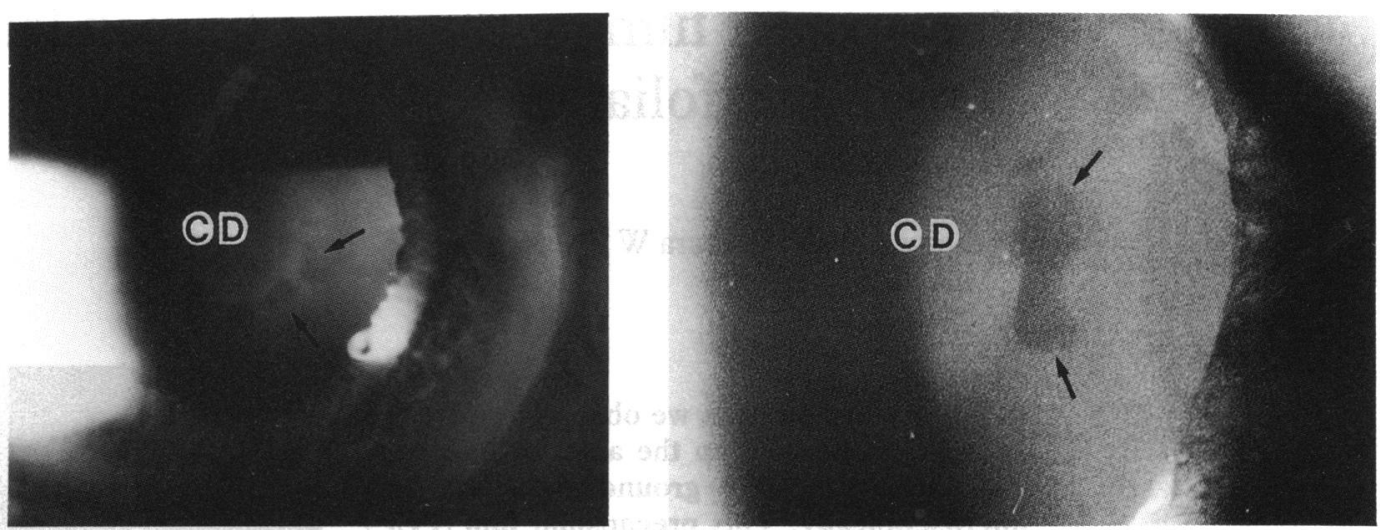

Figure 3 Anterior lens surface in two patients without evidence of pseudoexfoliative vegetations. Left: opacified precapsular film has several paracentral holes (arrows). Right: the central disc (CD) is denser, with a dumbbell-shaped hole at its edge (arrows).

not mention the presence of a diffuse film. A small number showed string-like shreds and nodules of rolled up PCF, and some had holes adjacent to the central area (Fig 3).

To determine the nature of the precapsular film we studied it on anterior lens capsules by light and electron microscopy.

\section{Material and methods}

Anterior lens capsules were obtained from 10 patients aged 65-80 undergoing capsulectomy for cataract extraction. These patients had previously given slit-lamp evidence of a PCF by showing peripheral grey striae on the midperipheral lens capsule, a more opaque central area, holes adjacent to the central area, or a combination of these findings. None had white vegetations characteristic of the granular phase of PSX. Specimens were fixed in $2.5 \%$ glutaraldehyde, dehydrated, and embedded in Mollenhauer's medium for transmission electron microscopy (TEM). Sections were stained with lead citrate and uranyl acetate. Capsulectomy speci-

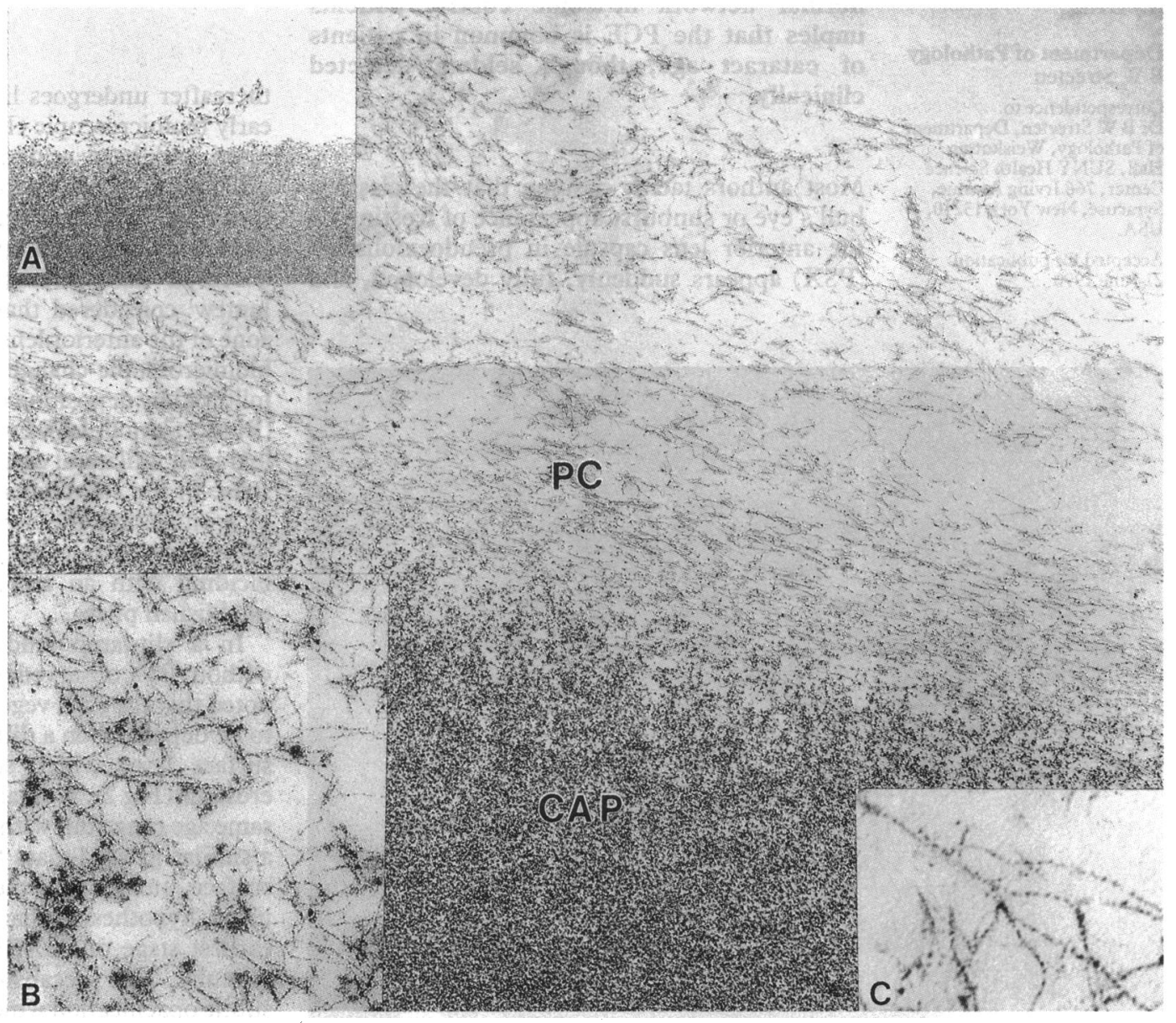

Figure 4 Thick precapsular film (PC) composed of a loose network of fibrils on a coarsely granular lens capsule (CAP). ( $\times 13$ 800.) Insert A: Peeling away of thinner PCF layer. $(\times 7900$.) B: Network of 3-6 nm fibrils at junction with granular capsule. $(\times 58000$.) C: $10-12 \mathrm{~nm}$ periodicity of thin fibrils at high power. $(\times 120400$.) 
mens were obtained in the same way from 10 control patients in this age range who showed no preoperative evidence of PCF or PSX. All the patients in the study were examined preoperatively by one of us (AJD) and about twothirds by both authors, residents, or other physicians, with concurrence on the clinical findings.

Four whole lenses noted to have PCF clinically were removed by intracapsular extraction. Three were examined by scanning electron microscopy after fixation in $2.5 \%$ glutaraldehyde, dehydration, and gold shadowing. The fourth lens was stained with monoclonal antibody to fibrillin, ${ }^{3}$ a protein associated with elastic microfibrils and zonules, by the immunoperoxidase method before fixation, as previously described. ${ }^{4}$ The capsule was then examined as a whole mount by light microscopy. Five lens capsules from normal eye bank eyes aged 16 to 83 years were similarly examined with fibrillin antibody as whole mounts.

Three lenses with clinical PSX disease were obtained by intracapsular extraction and fixed in $2.5 \%$ glutaraldehyde. Pieces from the central anterior capsule were prepared for TEM. Anterior capsules or whole capsules from three other PSX lenses were reacted with fibrillin antibody before fixation, and whole mounts were made for light microscopy, as described above.

\section{Results}

In all 10 capsulectomy specimens from patients in whom a PCF had been observed with the slitlamp preoperatively, transmission electron microscopy revealed a layer of fine fibrils on the anterior capsular surface (Fig 4). The layer was 0.5-6 $\mu \mathrm{m}$ in thickness, tending to fragment and separate from the capsule. Pigment granules or other organelles were rarely noted interspersed among the fibrils. The fibrils were variable in length and 3-6 $\mathrm{nm}$ in width, with a suggestive periodicity of 10-12 $\mathrm{nm}$ at high power (Fig 4C). They were irregularly arranged but generally parallel to the surface and blended into the superficial capsule. Where the layer was thickest, the immediately underlying capsule was usually coarsely granular (Fig 4). Five of the 10 control capsules showed a relatively smooth fibrogranular anterior surface, without visibility of individual fibrils (Fig 5A). The other five control capsules showed some evidence of a fine fibrillar layer, though it was generally much thinner, patchy in distribution, and associated with amorphous material (Fig 5B).

By TEM the central disc region of established PSX showed a fibrillar layer on the anterior surface which was partly composed of 3-6 nm fibrils similar to those in the PCF (Fig 6). The texture was, however, looser, and embedded among the finer fibrils were typical thick PSX fibres up to $35 \mathrm{~nm}$ in diameter with a macroperiodicity of $45-55 \mathrm{~nm}$. Also associated were some $8-10 \mathrm{~nm}$ fibrils with a $12 \mathrm{~nm}$ macroperiodicity, typical of zonular and other elastic microfibrils. The superficial capsule tended to be coarsely granular like that under the PCF.

By scanning electron microscopy (SEM) the lenses clinically showing a PCF had a superficial

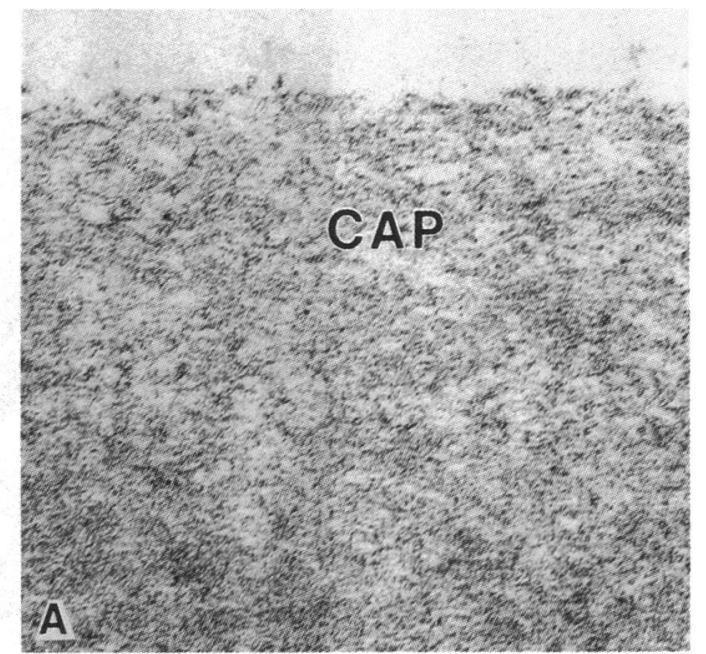

finely fibrillar layer of irregular outline (Fig 7), often having holes in it. Its edges were frequently peeled back or rolled into strings, which was doubtless artefactual, since it was not noted preoperatively, but showing the strong tendency of this thin layer to separate from the lens capsule. The fibrillar network was too fine to measure accurately by SEM.

Monoclonal antibody to fibrillin, an elastic microfibril-associated protein, was used to stain an entire lens capsule from a patient with a typical striated PCF. Besides staining zonular fibres and aging capsular inclusions in the usual fashion, the antibody bound to a fine network of loosely aggregated fibres on the anterior capsule central to the zonular insertion and blending into it peripherally. This network had an extensive but patchy distribution, tending to roll up at the edges or around eroded areas (Fig 8A).

By high power the fibres stained in a finely dotted pattern. In the mid zone the network 


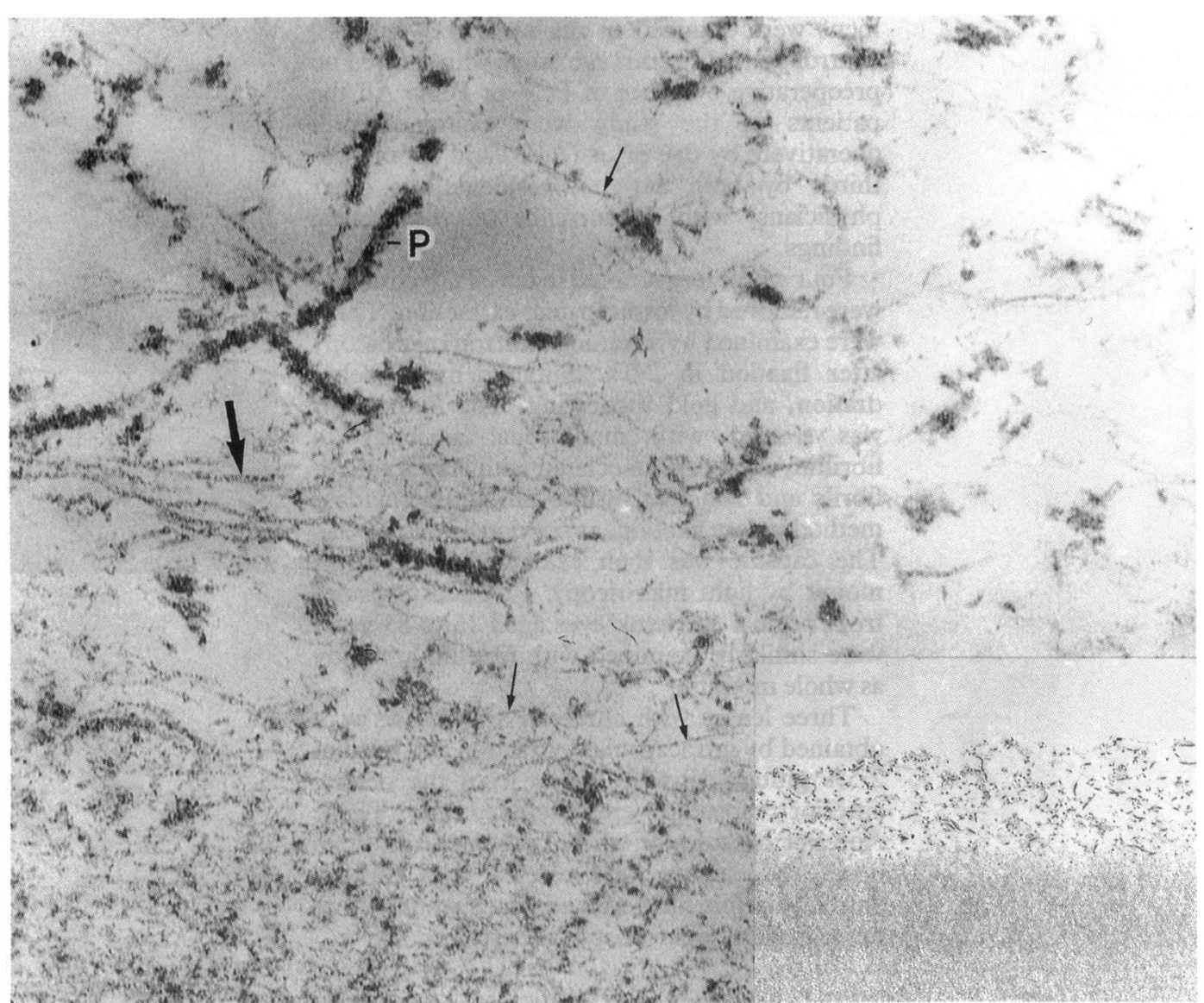

Figure 6 Fibrillar central disc on granular lens capsule in pseudoexfoliative disease. Fine 3-6 $\mathrm{nm}$ fibrils (thin arrows) intermingled with large coarsely banded $P S X$ fibres $(P)$, and 8-10 $\mathrm{nm}$ zonular-type periodic microfibrils (thick arrow). ( $\times 67$ 500.) Inset: Low-power view of this 0.3 um layer, $(\times 6150$.)

showed a radial striate pattern, approximating in number and location to the grey striae seen clinically (Fig 8B). A similar pattern was seen more diffusely in the PSX lenses (Fig $8 \mathrm{C}$ ), but there was also staining of the large PSX vegetations and deep fibrogranular zone. Control lenses had occasional wisps of fibrillin-staining network on the anterior capsule in patients over 40 years of age. The posterior capsule was unstained in all cases.

\section{Discussion}

The relucent or ground glass appearance of the superficial lens capsule we have noted in many older patients appears to correspond to the surface fibrillar network reported in the present paper, and called here the 'precapsular film'. The 3-6 nm fibrils composing most of the film are similar in size and morphology to the finer fibrils present in PSX vegetations, as well as on the surface and in the central disc area in established PSX disease. ${ }^{56} \mathrm{We}$ have shown that fibrillin, a glycoprotein closely associated with zonules and other elastic microfibrils, ${ }^{34}$ is present in all areas of PSX aggregates on the lens capsule. ${ }^{4}$

The staining pattern for fibrillin in the surface network of PSX lenses and a PCF lens with radial striae was very similar. It is not possible to say whether the 3-6 nm fibrils represent more than one fibre type in either condition, but the findings suggest that PSX material and the striated film share at least one common antigen besides the ultastructural similarity of some of their fibrils.

The clinical behaviour of PCF films such as peeling, and the development of holes often surrounding a denser central zone, also resemble patterns seen in the intermediate zone of established PSX disease (Fig 1). Moreover, the presence of an almost complete PCF film in some patients with established PSX (Fig 2) suggests that the film could be the initial stage of the disease process. Gifford described a 'frosting' of the anterior lens capsule, without differentiating granular and pregranular phases, as the earliest finding in PSX, before hole development forms an intermediate zone. ${ }^{7}$

In studying PSX among the Bantu, Bartholomew $^{1}$ found that a peripheral grey striation of the anterior lens capsule formed initially, and PSX vegetations were deposited subsequently. We have frequently noted such striations in Caucasians clinically on both PCF and PSX lenses, and show here similar striations with fibrillin antibody. They may represent imprints from radial folds on the posterior iris surface, possibly more marked in some races such as the Bantu. Visualising these peripheral grey striae appears to be one method for determining the presence of a PCF. Similar striae were reported by Schläpfer ${ }^{8}$ in $58 \%$ of glass factory workers, though in only $20 \%$ of clinic patients over 20 years of age. Bücklers' found some degree of striae in $77 \%$ of patients under 50 years of age 


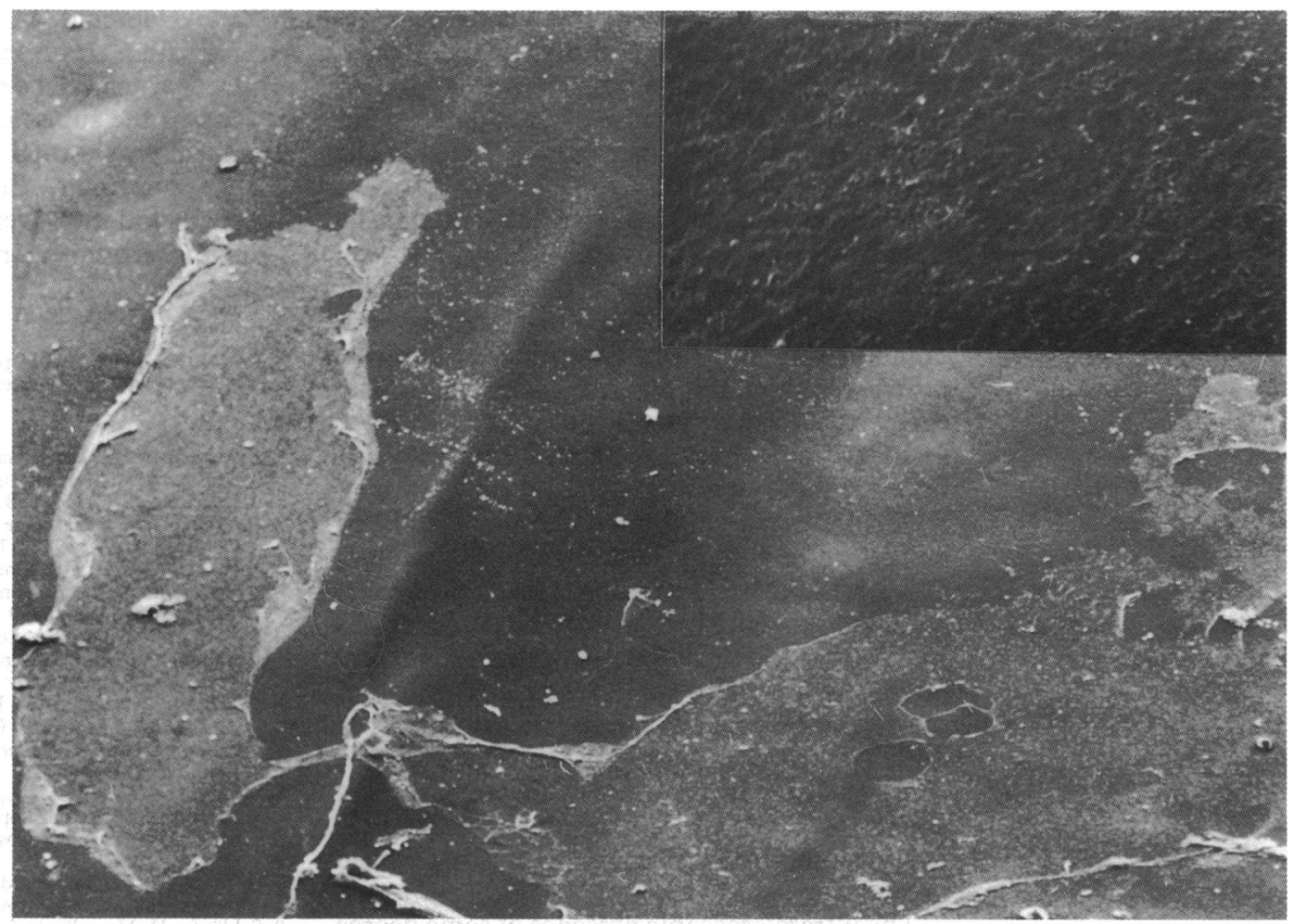

Figure 7 Scanning electron micrograph of anterior lens surface showing incomplete PCF eroded and rolled up at the edges, with string formation. From patient in Fig 3, right. $(\times 70$.) Inset: high power view of poorly defined fibrillar network. $(\times 2500)$.

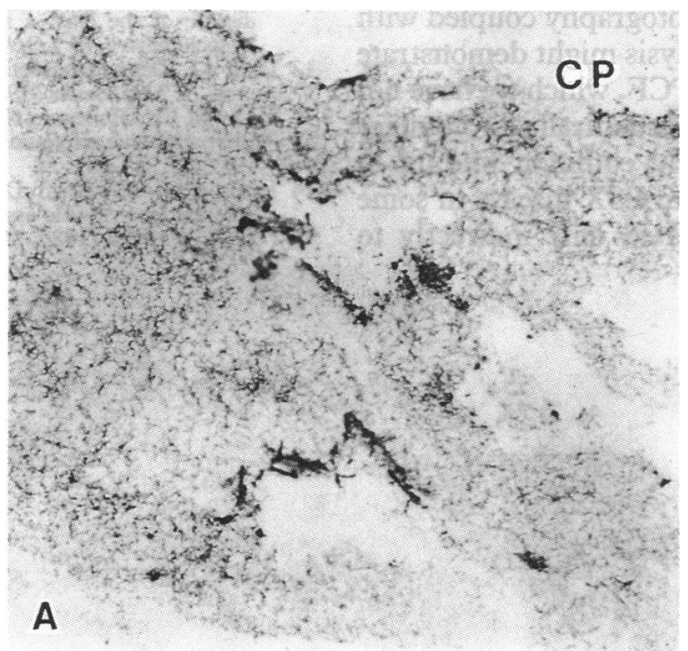

and concluded that they represented a physiological change, though suggesting that they could be a forerunner of senile capsular erosion ('Abschilferung'). None of these reports describe a diffuse PCF, which may be a more specific sign of PSX disease.

Jerndal ${ }^{2}$ found pigment deposits in the central and midperipheral zones of the anterior lens capsule at the initial biomicroscopic stage of pseudoexfoliation. He believed the pigment and subsequent exfoliation material derived from the iris pigment epithelium. We are aware that
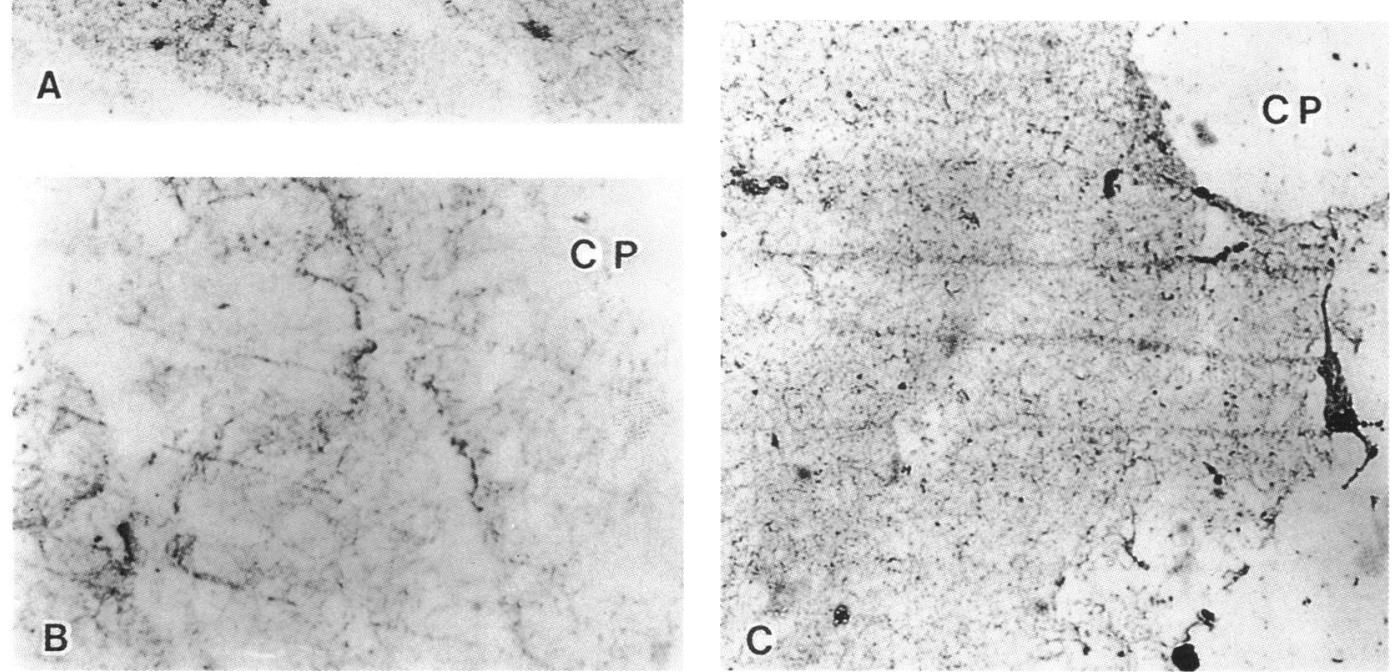

Figure 8 Anterior lens capsule reacted with fibrillin antibody. A: PCF shows a fibrillar network with focal holes on a nonstaining capsule $(C P)$. B: Faint radial striations in intermediate zone of a thin $P C F$. Capsule $(C P)$ is unstained. C: More compact surface network in PSX disease showing striations, peeling and string formation on non-staining capsule $(C P)$. (All immunoperoxidase, no counterstain $\times 500$.) 
pigmentary dispersion may be an early manifestation of PSX disease ${ }^{10}$ and that pigment cells, their granules, and other organelles are a variable component of PSX material,"11 12 but did not find any regular pattern of pigment deposition in our patients.

An idiopathic exfoliation of the lens capsule was recently described in seven elderly patients, ${ }^{13}$ one of whom had classical pseudoexfoliation. This condition appeared as a diaphanous membrane arising from the anterior lens capsule, protruding into the anterior chamber. It had some resemblance to the rolled up sheet-like material we depicted in the PCF by SEM, and what we have observed in some patients with PSX. An apparently unrelated transpupillary herniation of anterior lens capsule was recently noted in older peole in Somalia, strongly associated with climatic keratopathy but only weakly with established or classical PSX disease. ${ }^{14}$ It is of interest that a white opalescence or 'frosting' of the central capsule was considered a precursor of these protrusions. Lacking a knowledge of the ultrastructure of these two disorders we are unable to speculate further on their possible relationship to the precapsular film described here.

The ultramicroscopic presence of some degree of PCF in half of our control lenses, though undetected clinically, indicates that in the cataract age group such aggregates are frequent. A sparse or optically uniform film would be naturally difficult to see clinically. In such instances Scheimflug photography coupled with densitometric image analysis might demonstrate it. It is possible that the PCF, which we have not noted clinically or ultrastructurally in younger eyes, may be a relatively common finding in aging, only progressing to PSX disease in some patients, or perhaps related only indirectly to
PSX development. It is uncertain whether this surface film represents aberrant secretory products or material deriving from an aging lens surface, as the superficial capsule is often ultrastructurally abnormal as well. To understand this phenomenon, further knowledge about secretion and turnover of components in the zonular insertion-superficial lens capsular region is needed.

This work was supported in part by Research Grant EYO1602 from the National Eye Institute, National Institutes of Health.

1 Bartholomew RS. Pseudo-capsular exfoliation in the Bantu of South Africa. I. Early or pregranular clinical stage. $\mathrm{Br} \mathcal{F}$ Ophthalmol 1971; 55: 693-9.

2 Jerndal T. The initial stage of the exfoliation syndrome. Acto Ophthalmol (kbh) 1985; 173 suppl: 65-6.

3 Sakai LY, Keene DR, Engvall E. Fibrillin, a new 350-kD glycoprotein, is a component of extracellular microfibrils. $f$ Cell Biol 1986; 103: 2499-509.

4 Streeten BW, Gibson SA, Dark AJ. Pseudoexfoliative material contains an elastic microfibrillar-associated glycoprotein. Trans Am Ophthalmol Soc 1986; 84: 304-20.

5 Dark AJ, Streeten BW. Pseudoexfoliation syndrome. In: Garner A, Klintworth GK, eds. Pathobiology of ocular disease. A dymamic approach. Part B. M. Dekker, New York. 1982; Pg. 1303-20.

6 Streeten BW, Bookman L, Ritch R, Prince AM, Dark AJ. Pseudoexfoliative fibrillopathy in the conjunctiva. A relation to elastic fibers and elastosis. Ophthalmology 1987; 94 : 1439 49.

7 Gifford $\mathbf{H ~ J r}$. A clinical and pathologic study of exfoliation of the lens capsule. Am $\mathcal{F}$ Ophthalmol 1958; 46: 508-24.

Schläpfer H. Neue Beobachtungen über Glasmacherkatarakt. Klin Monatsbl Augenheilkd 1930; 85: 285-6.

9 Bücklers M. Die Strahlenkranztrubung ('Aureole'): eine physiologische Erscheinung an der Linsenvorderfläche. Klin Monatsbl Augenheilkd 1939; 103: 413-7.

10 Prince AM, Streeten BW, Ritch R, Dark AJ, Sperling M. Preclinical diagnosis of pseudoexfoliation syndrome. Arch Ophthalmol 1987; 105: 1086-2.

11 Dark AJ, Streeten BW, Cornwall CC. Pseudoexfoliative disease of the lens: a study in electron microscopy and disease of the lens: a study in electron microscop
histochemistry. $B r \mathcal{F}$ Ophthalmol 1977; 61: 462-72.

12 Dark AJ. Cataract extraction complicated by capsular glaucoma. Br f Ophthalmol 1979; 63: 465-8.

13 Cashwell FL, Holleman IL, Weaver GW, Gerrard H van R. Idiopathic true exfoliation of the lens capsule. Ophthalmology 1989; 96: 348-51.

14 Johnson G, Minassian D, Franken S. Alterations of the anterior lens capsule associated with climatic keratopathy. Brf Ophthalmol 1989; 73: 229-34. 